\section{Vapor-Liquid Equilibria of Model Alkanes}

\author{
J. Ilja Siepmann,", Sami Karaborni, and Berend Smit
}

\section{Koninklijke/Shell-Laboratorium Amsterdam (Shell Research B.V.) P.O. Box 3003, $1003 \mathrm{AA}$ Amsterdam, The Netherlands}

Received April 5, 1993

In this communication, the results of computer simulations of the vapor-liquid equilibria of $n$-pentane and $n$-octane are reported. We have used a novel Monte Carlo technique to simulate directly phase equilibria of chain molecules. The calculations were performed for a united-atom model using the Lennard-Jones parameter set of Jorgensen and co-workers. ${ }^{1}$ Comparison of the calculated phase diagrams with experimental data shows very good agreement, in particular for the shorter alkane.

Computer simulations have contributed to the general understanding of the behavior of chain molecules. The success of a simulation depends to a large extent on the judicious choice of molecular potentials. An example of a popular potential field is the OPLS model (optimized potentials for liquid simulations) which has been used to study systems ranging from simple alkanes ${ }^{1,2}$ and surfactants ${ }^{3,4}$ to polymers ${ }^{5}$ and proteins. ${ }^{6}$ It is important to realize that the OPLS model has been fitted to liquid properties of small molecules at room temperature. In practice, however, these models are used beyond the conditions at which they have been fitted. An ideal test of model potentials is the comparison with experimental phase diagrams. Unfortunately, determining a phase diagram is an extremely difficult task, ${ }^{7}$ in particular, for chain molecules.

Over the last few years there has been an impressive improvement in the development of simulation techniques for phase equilibria. Most noticeable is the introduction of the GibbsEnsemble Monte Carlo (GEMC) technique by Panagiotopoulos.,9 In the GEMC technique two separate boxes are utilized. Besides the conventional random displacement of particles, additional types of Monte Carlo moves are applied to ensure that the two boxes are in equilibrium, i.e., have equal temperature, pressure, and chemical potential. The particular advantage of the Gibbs ensemble is that if conditions are such that the system wants to phase separate, the simulation yields a vapor phase in one box and a liquid phase in the other. As a result, the coexistence properties can be determined directly. One of the Monte Carlo steps involves the swapping of a molecule from the vapor to the liquid box and vice versa. While this step is relatively easy for systems containing atoms or small molecules, it is virtually impossible to insert chain molecules into their liquid phase using the conventional techniques. To enhance the sampling of chain insertions we have used a novel technique based on the configurational-bias Monte Carlo method (CBMC). ${ }^{10,11}$ The random insertion is replaced with a scheme in which a chain molecule is inserted atom by atom such that conformations with favorable

\footnotetext{
I Present address: Department of Chemistry, University of Pennsylvania
} Philadelphia, PA 19104-6323.

(1) Jorgensen, W. L.; Madura, J. D.; Swenson, C. J. J. Am. Chem. Soc. 1984, 106, 6638-6646.

(2) Padilla, P.; Toxvaerd, S. J. Chem. Phys. 1991, 94, 5650-5654.

(3) Hautman, J.; Klein, M. L. J. Chem. Phys. 1989, 91, 4994-5001; Phys. Rev. Lett. 1991, 67, 1763-1766.

(4) Siepmann, J. I.; McDonald, I. R. Mol. Phys. 1992, 75, 255-259; Phys. Rev. Lett. 1993, 70, 453-456.

(5) de Pablo, J. J.; Laso, M.; Suter, U. W. J. Chem. Phys. 1992, 96, $2395-$ 2403. 1666

(6) Jorgensen, W. L.; Tirado-Rives, J. J. Am. Chem. Soc. 1988, 110, 1657-

(7) Allen, M. P.; Tildesley, D. J. Computer Simulation of Liquids; Clarendon, Oxford, 1987

(8) Panagiotopoulos, A. Z. Mol. Phys. 1987, 61, 813.

(9) Smit, B.; de Smedt, Ph.; Frenkel, D. Mol. Phys. 1989, 68, 931-950.

$0002-7863 / 93 / 1515-6454 \$ 04.00 / 0$

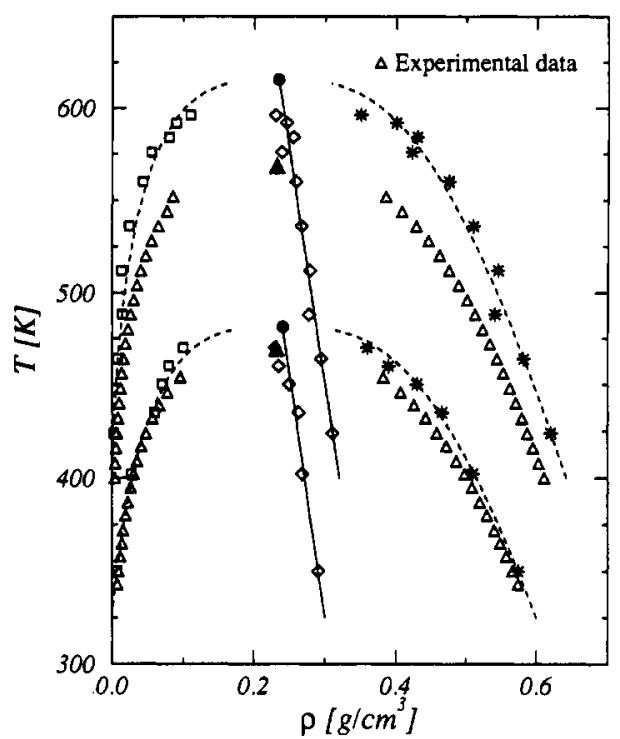

Figure 1. Vapor-liquid equilibria of pentane (lower curves) and octane (upper curves). $\Delta$ are the experimental data and the $\Delta$ is the experimental critical point. ${ }^{19} \square,{ }^{*}$ are the vapor and liquid densities as obtained from our simulations. $\diamond$ is the average of the liquid and vapor density. The solid lines are the fits to the scaling law and the rectilinear law. is the estimate of the critical point as obtained from these fits.

energies are preferred. To remove the resulting bias in the swapping step special acceptance rules were used. ${ }^{12}$ An intrinsic advantage of CBMC is that the conformational phase space is sampled very efficiently. 4,5,13 Large-scale conformational changes of the chain molecules are achieved by regrowing an entire chain molecule or part thereof. These moves avoid explicit crossing of conformational barriers.

The interaction model used to describe $n$-pentane and $n$-octane is based on the popular OPLS model of Jorgensen $e t$ al. ${ }^{1}$ The model makes use of the united-atom approach (i.e., methyl and methylene units are modeled as single pseudoatoms). Pseudoatoms in different molecules, and those belonging to the same molecule if separated by more than three bonds, interact which each other through a Lennard-Jones 12-6 potential using the OPLS parameters. The Lennard-Jones potentials were truncated at $11.5 \AA$, in line with the values used by Jorgensen during the fitting of the parameters. The pseudoatoms in a given chain are assumed to be connected by rigid bonds $\left(d_{\mathrm{cc}}=1.53 \AA\right)$; bond bending is modeled by a harmonic potential ${ }^{14}$ with the tetrahedral angles as equilibrium angle and with a force constant equal to $62500 \mathrm{~K} \mathrm{rad}^{-2}$; and changes in the torsional angles are controlled by the Ryckaert-Bellemans potential. ${ }^{15}$

The calculated vapor-liquid coexistence curves ${ }^{16}$ of $n$-pentane and $n$-octane are presented in the figure. Comparison with the

(10) Siepmann, J. I. Mol. Phys. 1990, 70, 1145-1158. Siepmann, J. I.; Frenkel, D. Mol. Phys. 1992, 75, 59-70. Siepmann, J. I. In Computer Simulation of Biomolecular Systems: Theoretical and Experimental Applications; van Gunsteren, W. F.; Weiner, P. K., Wilkinson, A. J.; Eds.; Escom Science: Leiden, 1993.

(11) Frenkel, D.; Mooij, G. C. A. M.; Smit, B. J. Phys.: Cond. Matt. 1992, 4, 3053-3076.

(12) Mooij, G. C. A. M.; Frenkel, D.; Smit, B. J.Phys.: Cond. Matt. 1992. 4. L255-L259.

(13) Siepmann, J. I;; McDonald, I. R. Mol. Phys. 1993, 79, 457-473. 3276.

der Ploeg, P.; Berendsen, H. J. C. J. Chem. Phys. 1982, 76, 3271-

15) Ryckaert, J, P - Bellemans, A Chem. Phys Lett. 1975, 30, 123-125.

(16) Six simulations at different temperatures were performed for a system containing 200 pentane molecules and 10 simulations for 160 octane molecules. Each simulation consisted of at least 7000 Monte Carlo cycles, of wbich the first 2000 were for equilibration. The convergence of the results was checked in many different ways (for the Gibbs ensemble see ref 9); in addition such measures as the fractions of gauche- and gauche ${ }^{+}$torsional angles and the end-to-end length were monitored. The statistical error in the simulation data is of the order of $5 \%$. 
experimental results shows that the OPLS model gives very good predictions for pentane and reasonable predictions for octane. At $423 \mathrm{~K}$ the coexisting densities of octane were also calculated by Laso et al. ${ }^{17}$ with similar results. From our simulations we determined the critical properties using the procedure described by Smit and Williams. ${ }^{18}$ For pentane the critical temperature

(17) Laso, M.; de Pablo, J. J.; Suter, U. W. J. Chem. Phys. 1992, 97, 2817-2819.

(18) Smit, B.; Williams, C. P. J. Phys.: Cond. Matt. 1990, 2, 4281.

(19) Smith, B. D.; Srivastava, R. Thermodynamics Data for Pure Compounds: Part A, Hydrocarbons and Ketones; Elsevier Science Publishers: Amsterdam, 1986.
$\left(T_{c}=481 \pm 5 \mathrm{~K}\right.$ ) is within $2 \%$ of the experimental value, and the critical density $\left(\rho_{c}=0.241 \pm 0.007 \mathrm{~g} / \mathrm{cm}^{3}\right)$ deviates by $4 \%$. For octane we obtain a critical temperature of $616 \pm 5 \mathrm{~K}$ which is within $9 \%$ of the experimental value, and a critical density of $0.234 \pm 0.008 \mathrm{~g} / \mathrm{cm}^{3}$ which is within $2 \%$ of the experimental result. If we recall that the OPLS parameters have been fitted for small alkanes at room temperature, this agreement is remarkable. It is noteworthy to mention that our simulation technique is not only limited to linear alkanes but can also be applied to other molecules such as branched alkanes, surfactants, and their mixtures for which experimental data might be difficult to obtain. 